\title{
Selecting Stakeholders for Requirements Elicitation in Agile Approach
}

\author{
Laith Obidat \\ Software Engineering Department \\ Isra University \\ Amman, Jordan
}

\begin{abstract}
Requirement's elicitations are the main stage in software development because the next stages or phases depends on it. Agile approach reduces cost and time into software development. The main problem in agile approach is the communications between stakeholders and developers. In this paper we will answer question: How to select right stakeholders to enhance communications between stakeholders and developers through proposed model to select stakeholders in agile approach to achieve strong communication led to enhance software development in agile approach.
\end{abstract}

\section{Keywords}

Requirements Elicitation; Stakeholder selection; Agile.

\section{INTRODUCTION}

In agile approach the most critical is the communication between developers and stakeholders because the acceptance on requirements based on users' opinions directly to success these communications should be selecting the right stakeholders that is one of main factors of software development success [1]. The agile approach development of software without formal requirements analysis and design phases through takes users' needs directly from stakeholders into many agile requirements practices in an organization. Such as face-to-face communications, review tests, extreme prioritization, and prototyping [2]. The stakeholders sometimes find difficult to comprehensive or trust the agile requirements process [2]. In this paper will be proposed model to select right stakeholders who are led to enhance communications between stakeholders to achieve right software development in agile approach. In new proposed model will identify the appropriate stakeholders, filtering, and prioritization based on some factors to select appropriate stakeholders then that lead to enhance communications in agile approach. This paper organized as follows. The following section provides the related work that is lead to new proposed model. Section III explains the methodology used that including new the proposed model.

Finally, section (4) explains summary and conclusion of the main results.

\section{RELATED WORKE}

To develop any system, a project team must elicit its requirements from the system's stakeholders. There are several definitions available for defining the concept of stakeholders. For example, stakeholders are defined as "any group or individual who can affect or is affected by the achievement of the organization objectives" [3].

From previous studies, it was in general classification stakeholders into four types: primary, secondary, external and extended stakeholders. Primary: their presence is necessary because it directly affects the outcome of the proposed system. The loss of any of the primary may affect the goals of the project and thus affected the development process. Secondary: are the people who will be affected by the project indirectly, in spite of their lack of participation in the process of development projects, but their goal to achieve control of their interests. Extended stakeholder: often are people who aim to provide assistance to stakeholders mentioned above to get to their visions. External: they are not part of the project team [3].

Due to the need for software development organizations to requirements that will evolve quickly and help reduce the time and cost it takes to develop software featured a new method called (agile). And are one of the newest and best ways to successful development and management of software compared with conventional methods [2].

One of the main advantages of this method is that the stakeholders in direct contact with the development and joint team constantly in the process of development where it can add or new requirements or modify the previous requirements, depending on the developer model of the previous phase as well as the change of priorities and ultimately get unusable and a short period of time a product [3].

There are several ways of communication between stakeholders and developers of the system, including: Face-toface communication, Iterative RE, Extreme prioritization, constant planning, Prototyping, Test-driven development, Reviews and tests [2]. This paper is intended to enhance communication between developers and stakeholders in Agile Requirement.

\section{METHODOLOGY}

The analysis was conduct in previous related work "selecting the right stakeholders for requirements elicitation: A systematic approach" ad previous related work which classified as a traditional approach modified in order to fit agile approach and how to employed it in agile project [3].

Figure 1 illustrates the new modified stakeholder selection framework. The framework consists of three essential stages; Identification, Filtering and Prioritization. Each stage comprises a set of elements. The first stage is Identification that uses project definition (goal, type and domain) to recognize stakeholders' types and roles (interaction and authority). This stage gathers as many potential stakeholders as possible that belong to the stipulated types and roles. As the quantity can be massive, the Filtering stage assesses stakeholders' knowledge and interests in order to gauge their efficacy and competency, also hire another additional filter suitable for agile was added to choose only who fit agile 
criteria. The Prioritization is the third stage, which finalizes the selection by measuring the chosen stakeholders' interpersonal skills. This is to justify their suitability [3]. The output of these three steps is a list of shortlisted candidates who are eligible to be involved in RE process. The following paragraphs elaborate the stages and elements in detail.

Stage 1 - Identification: At the beginning of any project, the main task is to set clear project definitions that include project goals and system descriptions (type and domain). The project goals specify what the business wants to achieve through the project while the system descriptions define the characteristics of the system to be built. The definitions lead to the recognition of which types of stakeholders (primary, secondary, external and extended) are required. Stakeholders have specific roles. They can therefore be categorized based on the roles that they are playing. Stakeholders may also be assigned to more than one role. Moreover, roles have degrees of importance. Some stakeholders' roles are more influential and significant than the others. These roles have higher chances of being considered in the next stage [3]. In order to determine which groups of stakeholders should be considered, they can be classified into the following classes:

- Mandatory (M) -Stakeholders that must be included or else the success of the system is threatened.

- Optional (O) - Stakeholders that are not necessarily selected. By neglecting their needs does not threaten the success of the system.

- Nice-to-have (N) - Stakeholders that do not influence the system's success if they are not selected.

Due to its importance, primary stakeholders are considered as $\mathrm{M}$ regardless the roles that they are playing. Secondary stakeholders may fall under M or O, depending on their roles' degrees of importance. External stakeholders are mainly $\mathrm{O}$ but they may become $\mathrm{N}$ if their involvement or roles towards the project are insignificant. Lastly, extended stakeholders fall under $\mathrm{N}$ as their contributions are quite minimal. The groups of stakeholders to be considered in the next stage are still massive after the classification.

Stage 2 - Filtering: In this stage, some forms of analysis should be conducted so that the selection can be made fairly. The analysis is based on three factors; knowledge, interest and suitability for agile. RE is known as the exchange of two essential knowledge between two parties; business domain knowledge from customers and software domain knowledge from developers. Interest is when a stakeholder's needs match with the project goals. On the other hand, suitability for agile means that the customer must fit agile conditions such as customer availability, customer "accepts" agile thinking, customer have the authority to make business decisions. One other important thing in this approach is that this stage is work in an iterative way, the iterations number ranged from 1 to 3 iterations per cycle depend on the resulted stakeholder, in details for example at the start of this filtering and as result of the previous stage only $\mathrm{M}$ class will be chosen, to participate in filtering stage and as a result for this stage list of possible stakeholders, but if there is need to enlarge the number of stakeholders then another second iterative will start and at this time only $\mathrm{O}$ class will be chosen to participate in filtering stage, this is because at the first iterative $M$ class already chosen and there is no need to expand to $\mathrm{N}$ class until the result of this filtering decided that. Certain measurement mechanisms have to be introduced to determine stakeholders' levels of; knowledge, interest and suitability for agile. The former is quite straightforward to measure, for instance, by assessing their educational background, past experience and job scopes. The latter however is a bit tricky as interest is normally implicit and psychological-influenced. Methods in psychology study such as interest inventory or personality testing may be adopted for this purpose. In short, this stage concerns assessing stakeholders' mental aptitude. The results are used to determine which stakeholders are eligible to proceed to the next stage.

Stage 3 - Prioritization: Projects have constraints which hinder project managers to include all possible stakeholders into a project. A kind of sorting process has to be established where certain aspects of the stakeholders enable them to be on top of the list. The sorting is called prioritization. Interpersonal skills are important to ensure an effective RE process. The skills therefore can be used as the final measures to qualify the selected stakeholders as the best possible participants. The skills include negotiation, collaboration and communication (written and oral). These three skills have to be considered holistically, which can be measured through predetermined tests. Some possible prioritization techniques that can be adopted include the ones that are normally employed in prioritizing requirements such as Analytical Hierarchy Process (AHP), Case-Based Ranking and Hierarchical Cumulative Voting.

\section{CONCLUSIONS AND FUTURE WORK}

In this paper we proposed new model to selecting appropriate stakeholders that leads to an enhance communication between customers and developers in agile approach. We suggestion using the proposed model because it including reselecting stakeholder to choose appropriate stakeholder who is selected to lead enhance communication between them. The proposed model can guide future work by applying this model to get correct results to prove it. 


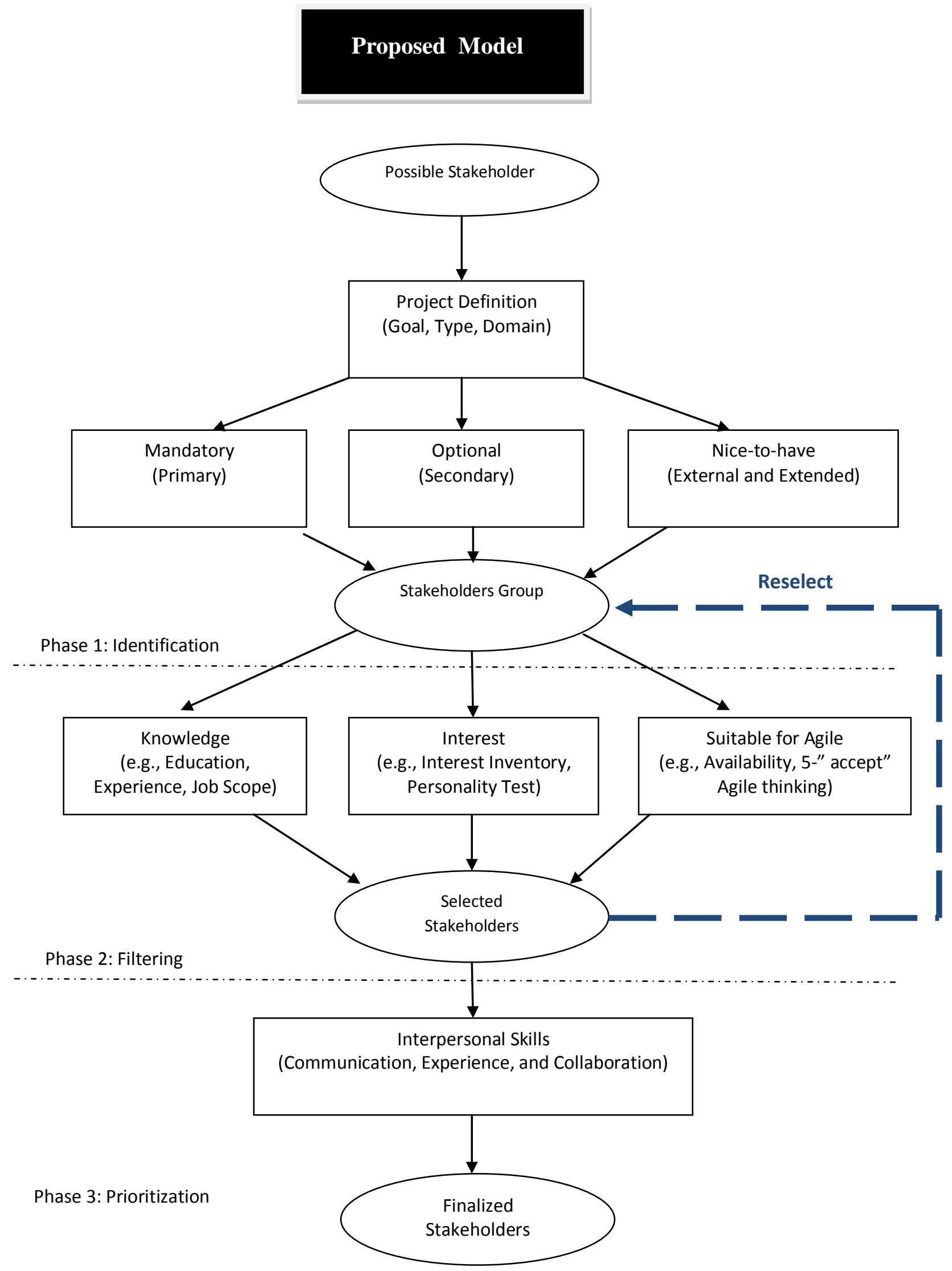

Fig 1: Proposed Model 


\section{REFERENCES}

[1] O. Preiss, and A. Wegmann, "Stakeholder discovery and classification based on system science principles," Proceedings of 2nd Asiapacific Conference on Quality Software, 2001, pp. 194-198.

[2] Lan Cao and Balasubramaniam Ramesh "Agile
Requirements Engineering Practices: An Empirical Study,” IEEE Computer Society, 2008, pp. 60-67.

[3] Rozilawati Razali, Fares Anwar "selecting the right stakeholders for requirements elicitation: A systematic approach," jornal of theoritical applied information technology, Vol. 33, 30th November 2011, pp. 250-257. 